\title{
Status quo wait times
}

I mprovements in wait times for priority procedures have levelled off across Canada as some provinces have failed to maintain previous gains in providing timely access to targeted surgeries, such as joint replacements, according to the Canadian Institute for Health Information (CIHI).

Roughly four out of five Canadians received priority-area procedures including hip and knee replacements, cataract surgery, hip fracture repairs and radiation therapy — within clinically recommended time frames for the second year in a row, CIHI says in its report, Wait Times in Canada - A Summary, 2012 (http://secure.cihi.ca /cihiweb/products/WaitTimesSummary 2012_EN.pdf).
But overall, there was "limited improvement" toward the target of providing such services in a timely manner to a minimum $90 \%$ of patients, except in the case of radiation therapy, which the majority (97\%) of patients received within the suggested 28-day time frame. In comparison, some $82 \%$ of patients received timely access to hip replacement and cataract surgery, while $79 \%$ underwent hip fracture repairs and $75 \%$ knee replacement surgery.

"It's difficult for provinces to get to that $90 \%$ mark because they're essentially chasing a moving target," says Kathleen Morris, director of health system analysis and emerging issues at CIHI. "About 50\% more knee replacements were performed last year than in 2004 , for example, and about $25 \%$ more cataract surgeries and hip replacements. That's a really staggering increase in volume in a short time frame."

The report shows patients wait the longest, and increasingly longer, for knee replacements. In British Columbia, Prince Edward Island (PEI) and New Brunswick, for example, the proportions of knee replacements performed within the recommended 182day time frame have dropped by at least 10 percentage points since 2009 to $67 \%, 55 \%$ and $53 \%$, respectively enough of a decline for CIHI to dub it a trend.

PEI also charted similar declines in the proportions of hip replacements and cataract surgeries that were performed within the recommended benchmark.

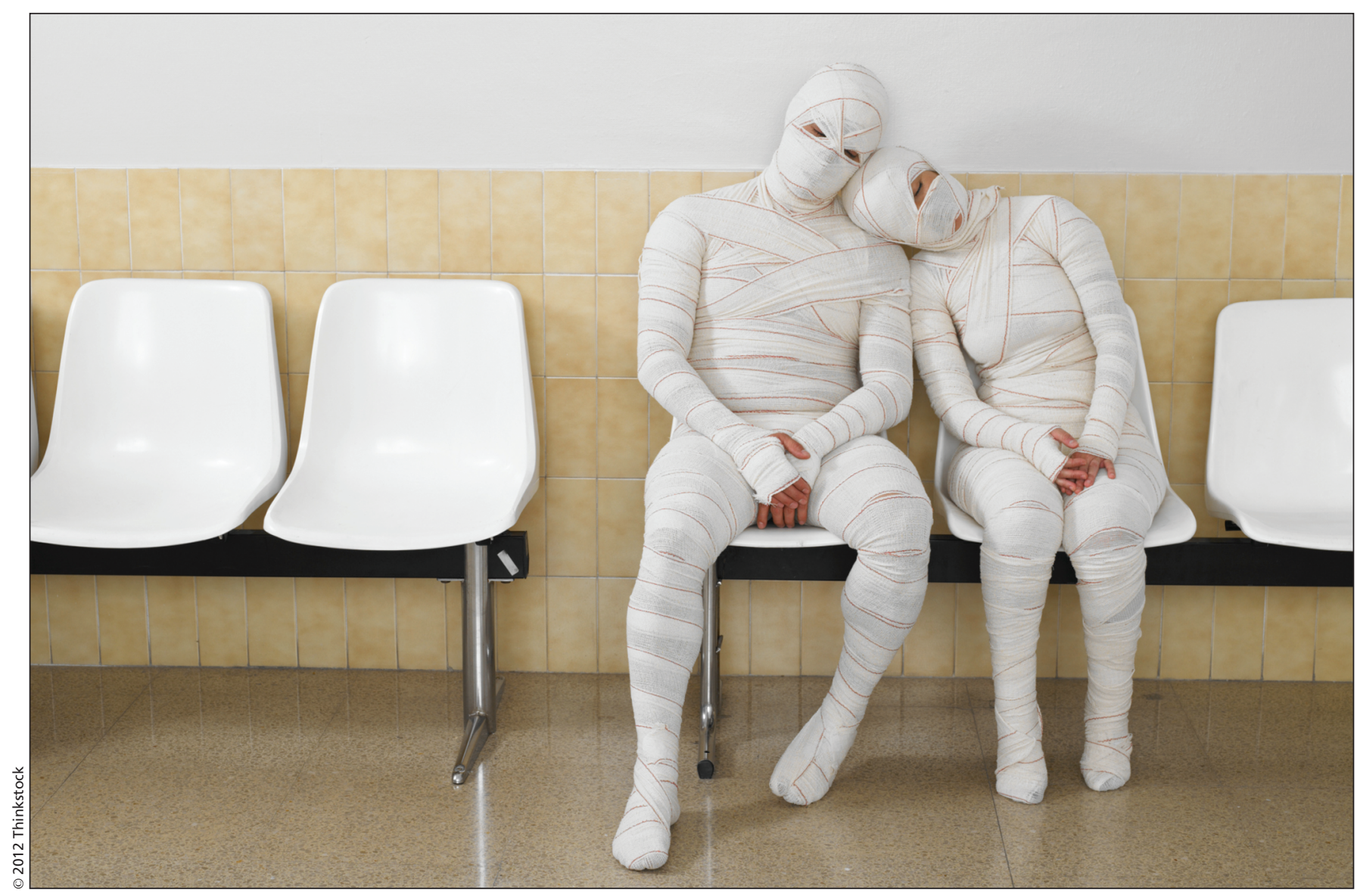

Providing $90 \%$ of Canadians with access to five designated priority medical procedures within medically recommended wait times may be unattainable, according to the Canadian Institute for Health Information. 
"If you look at knees specifically, some of [the decline may be due to] growth in health conditions like obesity and arthritis that drive the need for knee replacement surgery," Morris explains. "Surgeons are also more willing to do the procedure on younger patients, and some of that's due to the fact that the artificial joint itself is better now, so the replacements will last longer."

More generally, slower progress toward achieving the 90\% threshold for all five priority procedures may also indicate that Canada has already reaped the maximum benefit to be had from existing wait time reduction strategies, such as providing financial incentives to hospitals and surgeons to perform more of the procedures, she adds. "I think the health care system is now looking for some new strategies and one of them may be understanding more about the outcomes of surgery [in order to] predict those patients who will really receive the most benefit from surgery."

Such an approach, combined with a greater emphasis on preventive care, has met with success in international jurisdictions, although it would "need to be reviewed carefully," Morris says.

CIHI also urged more consistent provincial reporting of wait times for cardiac surgery.

The pan-Canadian benchmark for bypass surgery is 14-182 days, depending on how urgently that care is needed.
But "after seven years of cardiac wait times reporting, it is still unclear whether Canadians are receiving timely access to the cardiac surgery they need," the report states. That's because provinces typically only assess the percentage of patients receiving care within the longest benchmark (26 weeks), rather than "truly reflect the experiences of patients who have different requirements for treatment."

"To assess progress, we need a common approach to classifying patients into those urgency levels," Morris says. To date, though, "first guesses at what that might be haven't panned out." Lauren Vogel, CMAJ

CMAJ 2012. DOI:10.1503/cmaj.109-4163 